\title{
Hacia un breve glosario queer: algunas nociones acerca del género, la sexualidad y la teoría queer*
}

\author{
Hugo Córdova Quero*
}

Recibido: 23 de septiembre de 2019 • Aprobado: 15 de noviembre de 2019

\section{Resumen}

Este artículo busca corregir la necesidad de contar con un lenguaje común en los estudios queer. Tanto en la academia como en el activismo, se ha hecho evidente en los últimos años que, aunque usamos la misma terminología, no siempre nos entendemos. Cuestionar y desafiar las definiciones aprendidas y las prácticas arraigadas, a menudo por prejuicios, implica examinar los términos de la diversidad sexo-genérica. Este artículo no busca agotar la discusión ni hegemonizarla imponiéndose como paradigma. El objetivo, al contrario, es iniciar un diálogo que nos debemos en América Latina, en busca de una comprensión mutua de lo que queremos decir cuando usamos términos específicos de diversidad sexogenérica. Para ello, antes de examinar cada palabra, presento una breve revisión, como recordatorio, sobre lo que es la teoría queer. Soy consciente de que, desde la teoría queer, es imposible definir o encasillar conceptos y situaciones sin caer en su colonización y clausura. Por lo tanto, el tono del artículo gira en torno al diálogo y expresión de una forma particular de ver cada término sin considerar que su explicación aquí sea definitiva o final.

Palabras clave: activismo queer, cis-heteropatriarcalismo, cis-heteronormatividad, diversidad sexo-genérica, homonormatividad, teoría queer.

* Artículo de reflexión. Citar como: Córdova Quero, H. (2020). Hacia un breve glosario queer. Algunas nociones acerca del género, la sexualidad y la teoría queer. Análisis, 52(96), 95-121. Dol: https://doi.org/10.15332/21459169/5326

** Doctor en Estudios Interdisciplinarios en Religión, Migración y Etnicidad, Graduate Theological Union (GTU). Magíster en Teología Sistemática y Teorías Críticas (GTU). Magíster en Teología por el Instituto Superior Evangélico de Estudios Teológicos, Buenos Aires (Argentina). Profesor asociado de Teorías Críticas y Teologías Queer, Starr King School, Graduate Theological Union, Berkeley, California (Estados Unidos). Correo: hquero@sksm.edu. oRcid: https://orcid. org/0000-0001-9086-7756 


\section{Towards a brief queer glossary: some notions about gender, sexuality and queer theory}

Hugo Córdova Quero

\section{A bstract}

This article seeks to correct the need of having a common language in queer studies. Both in academia and in activism, it has become evident in recent years that, although we use the same terminology, we do not always understand. Questioning and challenging the definitions learned and entrenched practices, often due to prejudices, involves examining the terms of sex-gender diversity. This article does not seek to exhaust the discussion or hegemonize it by imposing itself as a paradigm. The objective, on the contrary, is to initiate a dialogue we owe ourselves in Latin America, in search of a mutual understanding of what we mean when we use specific terms of sex-gender diversity. To do this, before examining each word, I present a brief review, as a reminder, on what queer theory is. I am aware that from queer theory it is impossible to define or typecast concepts and situations without falling into their colonization and closure. Therefore, the tone of the article revolves around dialogue and expression of a particular way of looking at each term without considering that its explanation herein is final or definitive.

Keywords: queer activism, cis-heteropatriarchy, cis-heteronormativity, sexgeneric diversity, homonormativity, queer theory. 


\section{Vers un brève glossaire queer : quelques notions concernant le genre, la sexualité et la théorie queer}

Hugo Córdova Quero

\section{Résumé}

Cet article tente de répondre au besoin de disposer d'un langage commun dans les études queer. Que ce soit dans le monde académique ou dans l'activisme, il est évident que l'usage des mêmes termes ne garantit pas que l'on se comprenne. Questionner et défier les définitions apprises et les pratiques les plus enracinées implique remettre en question les mots qui expriment la diversité des sexes et des genres. Cet article ne prétend pas épuiser le débat ni s'imposer en paradigme. Le but consiste, au contraire, à entamer un dialogue que nous devons avoir en Amérique latin afin de nous comprendre sur ce que nous voulons dire quand nous utilisons certains mots. En ce sens, au lieu d'analyser chaque terme, je rappelle les lignes générales de la théorie queer, tout en étant conscient qu'il ne faut pas coloniser ou figer les concepts ou les situations. Par conséquent, cet article met en valeur le dialogue et l'expression d'une forme particulière de voir chaque terme sans songer à une définition péremptoire.

Mots clés: activisme queer, cis-hetero-patriarcat, cis-hetero-normativité, diversité sexuelle et de genres, homo-normativité, théorie queer. 


\section{Introducción}

Este artículo presenta algunos datos generales sobre la teoría queer y términos asociados. La primera parte ofrece una breve explicación sobre qué es la teoría queer, sin definir completamente algo que, desde su génesis, se ha resistido a ser definido. La segunda parte brinda definiciones sobre temas importantes como el género, la orientación sexual y la homosexualidad, entre otros. El propósito de este breve glosario queer no es ni agotar el significado de cada término ni convertirse en paradigma de su definición; por el contrario, busca ser una aproximación a la compleja realidad que cada término implica, con el fin de entablar un diálogo con distintas miradas sobre estos. Mucho menos se busca ofrecer una definición acabada sobre la teoría queer, sino más bien un punto de encuentro entre distintos modos de "hacer" esa teoría.

\section{¿Qué es la teoría queer?}

Las maneras en que las personas en sociedades europeas o norteamericanas ejercen su rol de género y las actuaciones de su sexualidad no son las mismas que en sociedades africanas, latinoamericanas o asiáticas. Más aún, esos modos tampoco han sido iguales en todos estos lugares, tanto en el pasado como en el presente, y seguramente en el futuro se presentarán también con cambios significativos. Algunas de estas maneras son colonizadas por otros modos que se autodenominan "más civilizados" o "más morales".

En la era de la globalización, las maneras en que la gente en sociedades europeas o norteamericanas ejercen su rol de género y las representaciones de la sexualidad han sido tomadas como modelo para otras partes del mundo, lo que también es aplicable para los movimientos lésbico-gay en esas latitudes. Este proceso ha sido tomado en países del sur global casi sin cuestionamientos, e internalizado a través de la noción colonialista de una sexualidad "normal", es decir, "universal". Todo esto es una operación de la hegemonía del sistema heterosexista aliado a un proyecto cultural colonialista. Pero ¿qué entendemos por heterosexismo? En su libro, Jung y Smith (1993) afirman que el heterosexismo está conectado a un sistema de apartheid:

Sugerimos que el heterosexismo no se funda primordialmente en miedos emocionales, odio, $\mathrm{u}$ otras respuestas viscerales a la homosexualidad. Sino que está enraizado en una inmensa constelación de creencias cognitivas sobre la sexualidad humana [...]. El heterosexismo [entonces] es análogo al racismo y al sexismo (pp. 13-14).

Por lo tanto, bajo la forma de un colonialismo sexual, el heterosexismo permea todas las dimensiones de la vida, del trabajo y de las relaciones, de la misma 
manera en que el racismo lo hace en la vida cotidiana. ${ }^{1}$ La teología no ha sido inmune a esta colonización heterosexista. Los cuerpos, las orientaciones sexuales, el género y las actuaciones de la sexualidad han sido colonizadas/os por dicho sistema, que ha penetrado todas las dimensiones de la vida cotidiana en maneras que muchas veces se tornan difíciles de contrarrestar. La teología muchas veces ha imbuido, legitimado o promovido aquellas dimensiones. Así, los cuerpos han sido la geografía de esta ocupación, el lugar donde el colonialismo heterosexual ha mantenido cautiva a la sexualidad y demarcado sus límites. En el cristianismo, cuerpos y sexualidad han sido terrenos prohibidos en pos de una supuesta "espiritualidad" que niega su doctrina central: la encarnación. Es decir, el cuerpo completamente humano de Jesús como lugar donde inhabitó la divinidad. La violencia de este proceso de negación es revelada en el proceso de normalización, también llamado heterosexualización. Nada que no sea encasillado dentro del binomio masculino/femenino heterosexual es dejado intacto. Por el contrario, debe ser cambiado, normalizado, normatizado, desterrado o demonizado.

Fue De Lauretis (1991) quien, en la introducción de un número especial de Differences: A Journal of Feminist Cultural Studies que ella editó, usó el término "teoría queer" por primera vez. Ese número especial se llamó “Queer Theory: Lesbian and Gay Sexualities"2. De Lauretis (1991) señala que el contexto del título de ese número fue producido con el fin de:

estar dispuestos a examinar, explicitar, comparar o confrontar las respectivas historias de supuestos y marcos conceptuales que han caracterizado a las auto-representaciones tanto de personas lesbianas y gays en Norteamérica como de gente de color y caucásicas hasta el momento: Desde allí, podríamos entonces pasar a la refundación o reinvención de los términos de nuestras sexualidades, para construir otro horizonte discursivo, otra forma de pensar lo sexual. (p. iv)

Así, De Lauretis (1991) ya marca una distancia entre los estudios queer y los estudios lésbico/gay, tal como continúa explicando:

La teoría queer transmite un doble énfasis sobre el trabajo conceptual y especulativo que implica la producción del discurso y la labor crítica necesaria de deconstruir nuestros propios discursos y sus silencios construidos. [...] El término "queer" yuxtapuesto a "gays y lesbianas" del subtítulo [de la revista en cuestión], pretende marcar una cierta distancia crítica respecto a esta última fórmula ya establecida y con frecuencia conveniente. Ya que la frase "lesbianas y gays" o "gays y lesbianas" se ha convertido en la manera estándar de referirse a lo que hace sólo unos pocos años solía ser simplemente "gay" (...) o, aún algunos años antes, "homosexual". (p.iv)

1 En términos lingüísticos, la conexión entre raza y sexualidad está relacionada con el origen de la palabra raza. Sobre esto, véanse los trabajos de Delany (1999) y Sterba (1997).

2 Teoría queer: sexualidades lesbianas y gays. 
En el mismo momento en que De Lauretis editaba esta obra, Butler publicaba El Género en Disputa (2007), un libro que pronto se convirtió en un recurso básico para lo que luego se llamaría "teoría queer". La autora no tenía esta intención, tal como lo reconoce en la segunda edición, publicada en 1999:

Hace diez años terminé el manuscrito de la versión inglesa de $E l$ género en disputa [...]. Nunca imaginé que el texto iba a tener tantos lectores, ni tampoco que se convertiría en una «intervención» provocadora en la teoría feminista, ni que sería citado como uno de los textos fundadores de la teoría queer. La vida del texto ha superado mis intenciones, y seguramente esto es debido, hasta cierto punto, al entorno cambiante en el que fue acogido. (Butler, 2007, p. 7)

En un tono casi ricoueriano ${ }^{3}$, Butler (2007) nos acerca a un hermoso reconocimiento de la propia trayectoria del texto. Este se basa en varios intelectuales franceses - quienes en los Estados Unidos han sido agrupados bajo la etiqueta de "teoría francesa" - : Michel Foucault, Jacques Lacan, Julia Kristeva, Sigmund Freud, Monique Wittig, Luce Irigaray, Simone de Beauvoiur, Jacques Derrida y Claude Lévi-Strauss (Butler, 2007). La autora piensa que esta situación es bastante extraña, ya que aquella etiqueta originada en Estados Unidos tiende a simplificar la diversidad y las diferencias que todas/os esas/os intelectuales tienen entre sí.

En todos los escritos sobre la teoría queer, es difícil encontrar una definición de lo que esta es. Jagose (1996), una teórica australiana, es quien quizás nos introduce de una manera clara y concisa en el complejo mundo de la teoría queer, con su libro Queer Theory: An Introduction, que tiene por objeto - entre otros - aclarar las diferencias y similitudes entre los términos homosexualidad, gay, lesbiana y queer. Al igual que en todos los escritos sobre la teoría queer, es difícil encontrar una definición de lo que esta teoría significa.

El término queer se puede traducir como "raro", y fue aplicado peyorativamente a las personas en el contexto anglosajón que no se conformaban con el heteropatriarcado. En la década de 1990, en Nueva York fue tomado por activistas como un término de reivindicación contracultural (Jagose, 1996). En la introducción de su libro, Jagose (1996) trata de delimitar las fronteras de lo queer de la siguiente manera:

En términos generales, lo queer describe los gestos o modelos analíticos que dramatizan las incoherencias en las relaciones supuestamente estables entre el sexo cromosómico, el género y el deseo sexual. [...] [Lo Q]ueer localiza y explota las incoherencias de esos tres términos que estabilizan la heterosexualidad. Demostrar

3 Paul Ricouer (1913-2005) fue un filósofo francés que influenció grandemente los estudios religiosos y teológicos con sus postulados sobre hermenéutica (interpretación) de los textos, especialmente de los textos sagrados. Ricouer (1994) afirma que la "relación" entre texto y referencia es "[...] la interpretación como reconocimiento de una modalidad de ser del mundo proyectada en el texto" (p. 65), precisamente a través de esa interpretación. Así, hay múltiples maneras de acceder a un texto, porque su constitución y sentido son variados. 
la imposibilidad de la sexualidad "natural," que pone en duda incluso términos que aparentemente no presentaban problemas tales como "mujer" y "varón". (p. 3)

Es decir, la teoría queer busca socavar los cimientos del heteropatriarcado, y toda persona que hace eso es parte del movimiento (Foucault, 1998; Sedgwick, 1998; Jagose, 1996; Halperin, 2003; Butler, 2007; Kemp, 2009; Marinucci, 2010). Para Jagose (1996), "lo queer es siempre una identidad en construcción, un espacio constante para llegar a ser" (p. 5).

Por lo tanto, lo queer no es sinónimo de LGBTI — sigla que representa al movimiento de lesbianas, gays, bisexuales, transgénero e intersexuales-, porque las personas heterosexuales que no adhieren al heteropatriarcado también son parte de la diversidad sexo-genérica. Así, Jagose (1996) afirma que "la convicción lésbico-gay en la autenticidad o incluso la eficacia política de las categorías de identidad y la suspensión queer de tales clasificaciones se energizan la una a la otra" (p. 5).

Por heteropatriarcado entendemos aquel sistema y organización social donde no solo prevalecen los criterios del machismo, sino que además solo se entienden como "normales", "naturales" o "humanas" las prácticas sexuales, afectivas, emocionales y románticas exclusivamente entre varones con mujeres, es decir, las heterosexuales.

Hay que decir que la teoría queer hoy en día está pasando por un momento que tiende a la normalización. Algunos estudiosos como Halperin (2003) han expresado su preocupación por esta "tendencia de normalización" de las instituciones académicas y los discursos hegemónicos. Por otro lado, la teoría queer parece ser replanteada en una especie de "limbo anglosajón" y no es capaz de ver cómo las negociaciones locales de identidad, las representaciones del género y la sexualidad, y el discurso pueden ser queer, pero no según la mentalidad norteamericana o nordeuropea. Esto es obvio en las diferentes concepciones sobre el matrimonio y la familia en distintas sociedades humanas que son invisibilizadas por la noción de la familia nuclear monógama, exportada desde occidente por los imperios dominantes entre los siglos XVI y XIX, como analizaré más adelante.

\section{Algunos términos necesarios}

Sería imposible agotar en este artículo todos los temas que se desprenden de la teoría queer y que impactan el modo en que entendemos aspectos relacionados con la sexualidad y con el género en el siglo xxi. Para efectos de contribuir y no confundir, en esta sección me centro en algunos términos básicos.

Recordemos que el género, la sexualidad y lo religioso se entrecruzan en la vida cotidiana de individuos y comunidades en nuestro contexto latinoamericano. El reconocimiento de este entrecruzamiento es, en realidad, un área emergente de análisis tanto para las ciencias sociales como para la teología y los estudios 
religiosos. Los discursos religiosos están intrínsecamente relacionados con lo social y lo cultural, y dan forma a la percepción sobre el género, la sexualidad, los cuerpos, la división heterosexual del trabajo y las expectativas de los roles de género. Navegar el complejo constructo de cada uno de estos elementos requiere un ejercicio de calibración conceptual. Es decir, los modos en que esos elementos han sido cargados de sentido refieren a negociaciones de poder y contextualidades que no siempre son comunes a cada interlocutor/a.

\section{Género}

Comencemos por afirmar que el género se trata de una "dimensión clave relacional" culturalmente informada y con "consecuencias para el posicionamiento social o cultural" tanto de mujeres como de varones (Indra, 2004, p. 2). El género ha sido "simplísticamente leído como 'mujeres' y no como relaciones de poder, privilegio y prestigio informado por nociones situadas de la masculinidad y la feminidad" (Indra, 2004, p. xiv). Basada en la noción de poder propuesta por Michel Foucault y los nuevos enfoques de género, Indra (2004) llega a la conclusión de que "existe un consenso creciente de que se debe dar más importancia a las diferencias de cultura, raza, clase, etnia, nacionalidad, y orientación sexual entre mujeres y varones" (p. 8). En otras palabras, el género es esencial para lograr que estas categorías se entrecrucen entre sí, con el fin de analizar las experiencias cotidianas vividas por las personas.

Como dije, el género no puede ser pensado como sinónimo de mujer. Eso sería dejar de lado la inmensa carga que se impone sobre mujeres y varones al reproducir la división heterosexual del trabajo. Varones y mujeres negocian los mandatos culturales de género al formar sus parejas o familias. El papel del cristianismo y su influencia son notables en la formación de familias en países donde el catolicismo romano u otra rama del cristianismo es mayoría. Esto está de acuerdo, de hecho, con el papel que el cristianismo ha jugado a lo largo de su historia. Las definiciones actuales y las expectativas de género, la división heterosexual del trabajo, o las normas relativas a la decencia y a la moral no fueron creadas en el vacío. Por el contrario, están construidas social, histórica y culturalmente.

A lo largo de su historia, en cada época el cristianismo ha asumido y negociado las construcciones socialmente sancionadas del género y la sexualidad, con el fin de moldear las expectativas sobre estos. El cristianismo fue capaz incluso de imponer al imperio romano sus propios puntos de vista después de ser proclamado como religión de Estado hacia el 380 e. c. (Brown, 1993). El catolicismo romano en América Latina ha determinado los modos en que varones y mujeres se relacionan entre sí. Aún hoy en día - pese a su decadencia numérica - impone su visión sobre muchos aspectos de las sociedades latinoamericanas.

En Occidente hemos heredado esa influencia cristiana, que también ha sido exportada e impuesta en otras sociedades, muchas de ellas colonizadas. Por lo tanto, la visión occidental sobre el género ha invisibilizado nociones autóctonas 
y ha impuesto modos particulares - y coloniales - de entender el género. La verdad es que no existe "una" noción de género sino muchas. Lo mismo puede decirse de las nociones de sexualidad, pareja, familia, amor, entre otras. Deconstruir la noción occidental de género es una tarea eminentemente necesaria para poder observar cómo la mentalidad occidental nos ha impuesto modos de "ser" que muchas veces nos resultan difíciles de cumplir. Precisamente eso es algo que la teoría queer busca asumir como mandato propio.

Butler (2007) ha dedicado su obra a deconstruir y cuestionar la noción occidental de género y, para ello, comienza afirmando que el género es una construcción basada en la ficción de una materialidad tácita:

El género es una construcción que reiteradamente disimula su génesis; el acuerdo colectivo tácito de actuar, crear y garantizar géneros diferenciados y polares como ficciones culturales queda disimulado por la credibilidad de esas producciones y por las sanciones que acompañan al hecho de no creer en ellas; la construcción nos «obliga» a creer en su necesidad y naturalidad. Las opciones históricas materializadas a través de distintos estilos corporales no son sino las ficciones culturales reguladas de forma punitiva, que alternadamente se personifican y se desvían bajo coacción. (p. 272)

El trabajo de Butler (2007) está basado en la presuposición de que las leyes que establecen el género se constituyen con base en prácticas repetitivas:

Las normas que gobiernan la identidad inteligible, o sea, que posibilitan y limitan la afirmación inteligible de un «yo», están parcialmente articuladas sobre matrices de jerarquía de género y heterosexualidad obligatoria, y operan a través de la repetición. ( $\mathrm{p}$. 282, énfasis en el original)

Esas prácticas repetitivas están cuidadosamente estructuradas con base en el pensamiento binario, que se traduce en códigos rígidos. Es precisamente dentro de las prácticas repetitivas que puede pensarse su disrupción, tal como continúa la autora:

Si las normas que gobiernan la significación no sólo limitan, sino que también posibilitan la afirmación de campos diferentes de inteligibilidad cultural, es decir, nuevas alternativas para el género que refutan los códigos rígidos de binarismos jerárquicos, entonces sólo puede ser posible una subversión de la identidad en el seno de la práctica de significación repetitiva (Butler, 2007, p. 282, énfasis en el original).

Por lo tanto, se hace necesario planificar los modos a través de los cuales esa interrupción del pensamiento binario se hace posible:

La principal tarea más bien radica en localizar las estrategias de repetición subversiva que posibilitan esas construcciones, confirmar 
las opciones locales de intervención mediante la participación en esas prácticas de repetición que forman la identidad y, por consiguiente, presentan la posibilidad inherente de refutarlas (Butler, 2007, p. 286).

Para Butler (2007), nuestros cuerpos promulgan una perspectiva del género, es decir, nuestros cuerpos actúan el género, y estas promulgaciones responden tanto a las determinaciones físico/corporales, como a los ideales que se originan en la propia promulgación. Además, Butler (2007) afirma lo siguiente:

No obstante, cuando se entiende la identificación como una incorporación o fantasía hecha realidad queda claro que la coherencia es anhelada, esperada e idealizada, y que esta idealización es efecto de una significación corporal. En otras palabras, actos, gestos y deseo crean el efecto de un núcleo interno o sustancia, pero lo hacen en la superficie del cuerpo, mediante el juego de ausencias significantes que evocan, pero nunca revelan, el principio organizador de la identidad como una causa. Dichos actos, gestos y realizaciones - por lo general interpretados-son performativos en el sentido de que la esencia o la identidad que pretenden afirmar son invenciones fabricadas y preservadas mediante signos corpóreos y otros medios discursivos. El hecho de que el cuerpo con género sea performativo muestra que no tiene una posición ontológica distinta de los diversos actos que conforman su realidad. (p. 266, énfasis en el original)

Es este acto performativo el que construye el significado del género y, por extensión, de nuestros cuerpos, como Butler (2007) continúa explicando: “Hay que tener en consideración que el género, por ejemplo, es un estilo corporal, un 'acto', por así decirlo, que es al mismo tiempo intencional y performativo (donde performativo indica una construcción contingente y dramática del significado)" (p. 271, énfasis en el original).

De esta manera, la performatividad deviene en la manera a través de la cual la construcción del género se expresa en la vida diaria. En la obra de Butler (2007), esto se ejemplifica mejor en el desempeño de transformistas (drag queens), que hiperactúan el género poniendo en evidencia todas sus aristas. Debido a esto, la autora afirma lo siguiente:

Si la verdad interna del género es una invención, y si un género verdadero es una fantasía instaurada y circunscrita en la superficie de los cuerpos, entonces parece que los géneros no pueden ser ni verdaderos ni falsos, sino que sólo se crean como los efectos de verdad de un discurso de identidad primaria y estable. (Butler, 2007, p. 267)

Así nos damos cuenta de que estamos completamente conectadas/os a la matriz heterosexual y condenados a repetir sus prácticas compulsivamente o a ser marginadas/os cuando no cumplimos con sus dictámenes. Sin embargo, Butler 
(2007) concluye que "la tarea no es saber si hay que repetir, sino cómo repetir $\mathrm{o}$, de hecho, repetir y, mediante una multiplicación radical de género, desplazar las mismas reglas de género que permiten la propia repetición" (p. 287).

\section{Identidad y fluidez}

Para la teoría queer, las categorías de identidades de género son múltiples y cambiantes, pues tienen como finalidad reflejar las diversas realidades que los individuos y las comunidades enfrentan en un mundo en constante fluidez, especialmente en relación con cómo se construye(n) nuestro(s) cuerpo(s) e identidad(es) (Córdova Quero, 2008). La manera como las identidades se forman y son comprendidas podría explicarse de varias formas. Básicamente, existen dos grandes tendencias: por un lado, la comprensión de la identidad de un individuo como esencia (es/existe), lo que responde a una visión más estática, a cuya tendencia llamamos esencialismo; por otro, encontramos la comprensión de que todas las identidades se construyen socialmente y devienen, llegan a ser, lo que conlleva una visión más fluida, a cuya tendencia llamamos constructivismo.

Butler (2002a) desarrolla mejor este punto en su libro Cuerpos que importan, donde analiza cómo el(los) cuerpo(s) se construye(n) socialmente y se materializa(n). Me gustaría centrarme en el cuerpo como un lugar para mostrar su contribución. Butler (2002a) comienza planteando una propuesta de pensamiento provocativa en favor de la comprensión de cómo sucede la materialidad/materialización del(os) cuerpo(s):

Yo propondría [...] un retorno a la noción de materia, no como sitio o superficie, sino como un proceso de materialización que se estabiliza a través del tiempo para producir el efecto de frontera, de permanencia y de superficie que llamamos materia. Creo que el hecho de que la materia siempre esté materializada debe entenderse en relación con los efectos productivos, y en realidad materializadores, del poder regulador en el sentido foucaultiano. (p. 28, énfasis en el original)

Para entender mejor cómo se produce esto, la autora también define la construcción como la reiteración de las normas:

Lo esencial estriba entonces en que la construcción no es un acto único ni un proceso causal iniciado por un sujeto y que culmina en una serie de efectos fijados. La construcción no sólo se realiza en el tiempo, sino que es en sí misma un proceso temporal que opera a través de la reiteración de normas: en el curso de esta reiteración el sexo se produce y a la vez se desestabiliza. (Butler, 2002a, p. 29)

En otras palabras, siguiendo su argumento, se muestra que el(los) cuerpo(s) también se construyen mediante la reiteración de las normas que producen su comprensión aquí y ahora. Al analizar la cuestión más a fondo, Butler (2002a) afirma lo siguiente: 
La formación de un sujeto exige una identificación con el fantasma normativo del "sexo" y esta identificación se da a través de un repudio que produce un campo de abyección, un repudio sin el cual el sujeto no puede emerger. Éste es un repudio que crea la valencia de la "abyección" y su condición de espectro amenazador para el sujeto. Por otra parte, la materialización de un sexo dado será esencial para la regulación de las prácticas identificatorias que procurarán persistentemente que el sujeto rechace la identificación con la abyección del sexo. (p. 20)

Prosiguiendo esta línea de pensamiento, la autora también especifica esto:

La formación, la elaboración, la orientación, la circunscripción y la significación de ese cuerpo sexuado no serán un conjunto de acciones realizadas en observancia de dicha ley; por el contrario, serán un conjunto de acciones movilizadas por la ley, la acumulación de citas o referencias y la acumulación de la ley que produce efectos materiales, tanto la necesidad vívida de aquellos efectos como la oposición vívida a tal necesidad. (Butler, 2002a, p. 33-34)

De esta manera, siguiendo a Lacan y su famoso escrito sobre el estadio del espejo y la formación del "yo", Butler (2002a) llega a la relación entre la materialidad y la identidad:

El proceso de esa sedimentación o lo que podríamos llamar la materialización será una especie de apelación a las citas, la adquisición del ser mediante la cita del poder, una cita que establece una complicidad originaria con el poder en la formación del "yo". ( $\mathrm{p}$. 38, énfasis en el original)

Siguiendo a Butler (2002a), es correcto afirmar que no podemos separar la manifestación de la identidad de su conexión con el(los) cuerpo(s). En otras palabras, nuestro(s) cuerpo(s) es(son) necesario(s) para nuestra forma de visualizarnos/ desplegarnos a nosotras/os mismas/os en el mundo. Sin embargo, estos cuerpos no se limitan a performatividades fijas/estáticas; más bien, varían de acuerdo con múltiples experiencias que también modificarán la forma en que las identidades se construyen. Tanto la interacción como la performatividad permiten el poder del sistema heteropatriarcal de moldear esas identidades, pero al mismo tiempo hacen posibles interrupciones y resistencias a la heteronormatividad. Recordar esto es importante, con el fin de abrir espacios para la recepción de las experiencias de toda la diversidad sexo-genérica, especialmente para las personas bisexuales, transgénero e intersexuales, que a menudo quedan al margen de la diádica "lésbico-gay".

\section{Homosexualidad}

El término homosexualidad fue creado en 1869 por el escritor austrohúngaro Károly Maria Kertbeny (Elliot, 2003). Esto no significa que antes de ese momento 
no haya habido personas que se sentían atraídas por otras personas de su mismo sexo. Lo que sucedió con la creación del término es que se comenzó a hablar de un grupo específico - los homosexuales - desde una posición negativa y medicalizada, tal como lo afirma Foucault (1998):

El homosexual del siglo xIx ha llegado a ser un personaje: un pasado, una historia y una infancia, un carácter, una forma de vida; asimismo una morfología, con una anatomía indiscreta y quizás misteriosa fisiología. (p. 56)

En ese momento también surgió la idea de la homosexualidad como "enfermedad". Hasta la década de 1970, la Asociación Estadounidense de Psiquiatría (APA, por sus siglas en inglés) afirmaba que ser "homosexual" era estar "enfermo". En realidad, el término que la APA usaba era enfermedad mental. Sin embargo, a partir de 1973, la APA dejó de afirmar esto y quitó la homosexualidad de su Manual diagnóstico y estadístico de los trastornos mentales (APA, 2014) - el catálogo de enfermedades mentales conocido como DSM, por sus siglas en inglés-, por entender que no había nada de "enfermedad" en ella (Drescher, 2015). Esta decisión, que terminó con la estigmatización de muchas personas sufrida con anterioridad a 1973, no fue tomada livianamente, sino que se consultaron expertos de distintas áreas de las ciencias médicas, sociales y psicológicas para arribar a la misma conclusión.

Si bien la APA — que influye profundamente en los tratamientos psicológicos y psiquiátricos en todo el mundo, incluidos los países de América Latina - tomó esa decisión, en las sociedades contemporáneas la idea de la homosexualidad como "enfermedad" no se ha borrado completamente del imaginario colectivo. Aún hoy, algunas personas - y también algunas instituciones sociales o religiosas - siguen afirmando que la homosexualidad es una enfermedad, sin tomar en cuenta la evidencia científica que dice lo contrario. Asimismo, muchos profesionales siguen sosteniendo que las terapias reparadoras pueden "curar" a las personas. Esto es científica y estadísticamente erróneo. Las personas que han pasado por estas terapias no han sido "curadas", sino torturadas, y se les ha cercenado la posibilidad de ser felices, dejándoles secuelas que les afectan para toda la vida (Haldeman, 1994; Schidlo \& Schroeder, 2002).

Es esta idea social tan arraigada a la que se enfrentan las personas no-heterosexuales. Sus vidas se ven afectadas, juzgadas o condenadas por esa falsa concepción. Por lo tanto, un primer paso es desterrar la idea de que las personas de la diversidad sexo-genérica están "enfermas", para luego afirmar que son personas humanas con sentimientos y con deseos particulares, pero que esos sentimientos y deseos no son ni negativos ni - mucho menos - destinados a ser condenados.

\section{Orientación sexual}

Seguramente en muchos lugares de América Latina escucharemos que ser parte de la diversidad sexo-genérica es no ser "normal". Mucha gente dice que estas 
personas están "enfermas" y "necesitan ser curadas". Seguramente al leer estas líneas no podemos evitar preguntarnos si esto es cierto. La respuesta es ¡no! Ser parte de la diversidad sexo-genérica no es estar ni enferma/o ni tener algo "contagioso". Por el contrario, es un hecho más de la diversidad presente en la vida de este planeta, incluida la humanidad. Es usual que la mayoría de las personas se relacionen sexualmente o afectivamente con personas del género opuesto, pero esa no es la única realidad en la especie humana; tampoco lo es en el reino animal. La diversidad sexo-genérica forma parte de la riqueza de la biodiversidad de este planeta, en el que la humanidad es solo un integrante más.

La orientación sexual no es algo que se elige, ni tampoco es algo que se forma genéticamente. Entendemos por orientación sexual la atracción emocional, romántica o el deseo sexual que algunas personas tienen por personas de distinto o de su mismo sexo o por ambas. Existen muchos prejuicios sobre por qué una persona tiene una orientación sexual determinada, incluyendo las falsas concepciones acerca de que las personas no-heterosexuales provienen de "hogares con problemas" o con un trasfondo de "mal desarrollo psicológico". No hay evidencia científica que apoye estos prejuicios. Las personas de la diversidad sexo-genérica son hijas e hijos de distintos tipos de familias, independientemente de su condición económica, clase social, etnicidad, cultura o contexto geográfico, entre otros aspectos. Simplemente estas personas son parte de la multiplicidad y riqueza humana.

\section{Roles de género y la división heterosexual del trabajo}

Las maneras en que las personas en sociedades europeas o norteamericanas definen el género, ejercen las expectativas de los roles de género y o entienden la división heterosexual del trabajo no son las mismas que en sociedades africanas, latinoamericanas o asiáticas. La expresión "expectativas de roles de género" se refiere a "la anticipación del desempeño dado por el actor social y por los demás en función de su posición sociocultural y económica, considerando la división predominante del trabajo entre los géneros" (Sudha, 2000, p. 63). Del mismo modo, el término división heterosexual del trabajo designa el "arreglo natural [tradicional] que constituye la base de toda especialización económica y estructuración social que conduzca a la formación de grupos de parentesco y de la familia" (Franke-Watson, 1999, p. 1299). Yamanaka (2003) ha descrito la división heterosexual del trabajo en términos donde padre equivale a "sostén de la familia" y madre implica "criar a los hijos y realizar actividades domésticas" (p. 169).

Cabe destacar que en todas las sociedades los modos de entender y ejercer estos aspectos tampoco han sido - ni son - iguales ni en el pasado ni en el presente, y seguramente tampoco lo serán en el futuro. Al mismo tiempo, es necesario aclarar que algunas de estas maneras son el resultado de imposiciones coloniales llevadas a cabo por imperios que se autodenominaron como más "civilizados" o más "morales" respecto de sus colonizados. En la era de la globalización, las 
maneras en que la gente en sociedades europeas o norteamericanas definen el género, ejercen las expectativas de los roles de género y o entienden la división heterosexual del trabajo han sido tomadas como el modelo para otras partes del mundo, lo que también es aplicable para los movimientos lésbico-gays en esas latitudes.

\section{El proceso de normalización}

El proceso de normalización lleva consigo la semilla de lo que Foucault (2002), en su libro Vigilar y Castigar, ha llamado la mirada normalizadora. Foucault (2002) analiza esta idea de la siguiente manera:

En suma, el arte de castigar, en el régimen del poder disciplinario, no tiende ni a la expiación ni aun exactamente a la represión. Utiliza cinco operaciones bien distintas: [1] referir los actos, los hechos extraordinarios, las conductas similares a un conjunto que es a la vez campo de comparación, espacio de diferenciación y principio de una regla que seguir. [2] Diferenciar a los individuos unos respecto de otros y en función de esta regla de conjunto - ya se la haga funcionar como umbral mínimo, como término medio que respetar o como grado óptimo al que hay que acercarse. [3] Medir en términos cuantitativos y jerarquizar en términos de valor las capacidades, el nivel, la "naturaleza" de los individuos. [4] Hacer que juegue, a través de esta medida "valorizante", la coacción de una conformidad que realizar. [5] En fin, trazar el límite que habrá de definir la diferencia respecto de todas las diferencias, la frontera exterior de lo anormal (la "clase vergonzosa" de la Escuela militar). La penalidad perfecta que atraviesa todos los puntos, y controla todos los instantes de las instituciones disciplinarias, compara, diferencia, jerarquiza, homogeiniza, excluye. En una palabra, normaliza. (pp. 187-188)

El proceso de normalización responde al colonialismo de las relaciones de la economía y el poder. Irigaray (1985), en su obra El sexo que no es uno, denuncia la transformación de los cuerpos en objetos estéticos a través de una lógica que transforma producciones simbólicas en producciones materiales. La autora asevera lo siguiente: “El valor de las producciones simbólicas e imaginarias se superpone a, e incluso es sustituido por, el valor de las relaciones de (re) producción material, natural y corporal" (p. 171).

El proceso de normalización ha sido un instrumento de la heterosexualidad compulsiva para vigilar y controlar las diferentes representaciones de la sexualidad y del género que no encajan en sus definiciones obtusas. Se asume una idea de la normalidad que es ficticia. Davis (1997), en su artículo "Constructing Normalcy: The Bell Curve, the Nobel, and the Invention of the Disabled Body in the Nineteenth Century" ${ }^{\prime}$, ofrece una historia de esta noción de la normalidad

4 La construcción de la normalidad: la curva de Bell, el Nobel, y la invención del cuerpo con discapacidad en el siglo xIx. 
corporal. Reconoce que Adolphe Quetelet (1796-1847), un estadista francés, es considerado el padre de la concepción moderna de la norma o la media. Quetelet desarrolló este concepto después de su aplicación de la "ley del error" astronómico "a la distribución de las características humanas como la altura y el peso" (Davis, 1997, p. 11). De sus estudios, desarrolló la idea del ser humano promedio (l'homme moyen) como el ser humano abstracto a través del cual la vida promedio de toda la humanidad tiene que ser medida. Aún más, era deseable llegar a ser como este ser humano promedio (Davis, 1997).

Sin embargo, lo normal genera una gran categoría de exclusión. Las personas queer han experimentado este proceso de normalización de diferentes maneras en la sociedad y en las instituciones religiosas, tanto a través de actitudes discriminatorias o por medio de etiquetas utilizadas para definir la diversidad sexo-genérica. El problema no es solo que las etiquetas crean/construyen a alguien como diferente, sino que también aquellas personas que son etiquetadas como diferentes terminan creyendo que son diferentes. La idea de la desviación es una consecuencia de la noción de normalidad. Sir Francis Galton hizo algunas revisiones a esta noción:

Lo que estas revisiones de Galton significan son un intento de redefinir el concepto de lo "ideal" en relación con la población en general. En primer lugar, la aplicación de la idea de una norma para el cuerpo humano crea la idea de la desviación o un cuerpo "desviado". En segundo lugar, la idea de una norma empuja la variación normal del cuerpo a través de una plantilla más estricta guiando la forma en que el cuerpo "debe" ser. En tercer lugar, la revisión de la "curva normal de distribución" en cuartiles, clasificados en orden y así sucesivamente, crea un nuevo tipo de "ideal." Este ideal estadístico es diferente del ideal clásico que no contiene ningún imperativo de la norma, que luego se complementa con la noción de progreso, la perfectibilidad humana, y la eliminación de la desviación, para crear una visión dominante, hegemónica de lo que el cuerpo humano debiera ser. (Davis, 1997, p. 17)

A través del concepto de normalidad en la Ilustración, las personas dentro de las sociedades occidentales han sido privilegiadas como la medida de la normalidad y dan sentido a la realidad, lo cual coloniza y califica al resto de las culturas y sociedades en el mundo no-occidental. La sexualidad, así como todas las dimensiones de la vida humana, sufre las consecuencias de esta noción aún hoy en día (Goffman, 1986).

Vemos así cómo el análisis necesita no solo hacerse a nivel de los sistemas macrocósmicos en contextos poscoloniales, sino también a nivel de los aspectos microcósmicos de las relaciones humanas, como el género y la sexualidad. Hay una conexión íntima y directa entre lo que sucede en el nivel macroscósmico, ya sea en las estructuras de la economía o en el nivel de las construcciones sociales, y en el nivel microcósmico, ya sea en nuestras relaciones cara a cara o en las performatividades del cuerpo, la mente, el género y la sexualidad. 


\section{La noción de familia nuclear heterosexual}

La noción occidental contemporánea de la familia nuclear heterosexual y monógama es producto de la modernidad burguesa, cuya formación se produjo en tiempos de la primera revolución industrial capitalista europea (ca. 1750). Así, familia y heterosexualidad se unieron indisolublemente. Por ello, Butler (2002b) se pregunta si el matrimonio es siempre heterosexual y concluye que "el campo sexual se circunscribe de tal manera que la sexualidad ya es pensada en términos de matrimonio y el matrimonio ya está pensado como la adquisición de la legitimidad [heterosexual]" (p. 18).

El papel del cristianismo y su influencia son notables en la formación de familias en países donde el catolicismo romano, las iglesias evangélicas u otra(s) rama(s) de esta expresión religiosa es/son mayoría. Esto está relacionado con el papel que el cristianismo ha jugado a lo largo de su historia. Las definiciones actuales de la familia nuclear y las expectativas de género, la división heterosexual del trabajo, o las normas relativas a la decencia y a la moral no fueron creadas en el vacío. Por el contrario, están construidas social, histórica y culturalmente, en espacios donde el cristianismo ha tenido y tiene aún hoy un rol central como expresión identitaria (Córdova Quero, 2013).

Aprobada por el cristianismo - que acompañó el surgimiento del capitalismola noción de familia nuclear heterosexual llegó a ser entendida socialmente como "sancionada divinamente". Al final, esta construcción no podía ser cuestionada, ya que fue tomada como "natural" en todo el mundo occidental. Pronto, tanto el catolicismo romano como el protestantismo expandieron esta idea al resto del mundo, tanto a través de sus clérigos - ministros, sacerdotes y monjas - como de laicos, quienes reprodujeron la ideología heteropatriarcal entre los nuevos conversos. En consecuencia, la noción de familia nuclear moderna se convirtió en el modelo para todas las familias más allá del ámbito exclusivamente occidental (Córdova Quero, 2010). Esto se reproduce en las lecturas sobre lo divino, lo que retroalimenta la divinización de modelos humanos de género, sexualidad y de relacionamiento sexuales o afectivos (Córdova Quero, 2011).

De hecho, un recuento histórico muestra que las nociones particulares sobre la construcción del género, la sexualidad y los cuerpos, tanto en Europa occidental como en Norteamérica, fueron adoptadas por el cristianismo después de la Reforma protestante. Sobre esto Nussbaum (1997) afirma lo siguiente:

Incluso la incursión más superficial en antropología comparada e historia social deja en claro que la familia 'nuclear' como unidad encabezada por una pareja heterosexual, que habita en su propia casita privada, y comprometida con su intimidad el uno para con el otro y con el bienestar de sus hijos está tan lejos de ser 'natural' que apenas ha existido fuera de Europa Occidental y América del Norte luego de la Reforma protestante. (p. 31) 
Es importante tener en cuenta que existen culturas que sostienen la poligamia como construcción "natural" de familia y que deben luchar por su derecho a coexistir en contextos donde la "familia nuclear heterosexual" es hegemónica. Este es el caso, por ejemplo, de las personas in/migrantes. En sus países de residencia, esto no es un gran problema, pero cuando personas de estas culturas migran hacia otros lugares se revela el conflicto con la noción occidental de familia nuclear. Las y los in/migrantes llevan consigo esas nociones al país receptor junto con otros comportamientos culturales y sociales aprehendidos en su lugar de origen, enfrentando la cens ura del país receptor. Esto incluso es visto negativamente en discursos académicos. Ticktin (2008) estudia el caso del feminismo francés, que muchas veces juzga las costumbres de otros pueblos con base en estereotipos - por ejemplo, islam como "símbolo de violencia" (p. 871)-, lo que concuerda con discursos que tienden a la antiinmigración y al control riguroso de las fronteras.

Navegar la propia cultura en tierra extraña implica enfrentar prejuicios y discriminaciones largamente arraigadas en la sociedad receptora. Lo mismo puede decirse de individuos provenientes de sociedades matriarcales o de sociedades con una división heterosexual del trabajo distinta a la prevalente en Occidente (Córdova Quero, 2016). El cristianismo - por ser una religión universal - a menudo encuentra esta situación en sus comunidades de fe, principalmente en aquellas que se encarnan en un ámbito intercultural. Tanto la sociedad civil como las organizaciones religiosas no siempre dan la bienvenida a quienes son consideradas/os como "otros", noción que muchas veces conlleva una mirada orientalista y condescendiente con aquellas personas de otras culturas. El género, la sexualidad y las nociones de familia están firmemente arraigadas en lo cultural.

\section{Cisgeneridad}

Desde la década de 1990, ha habido una creciente toma de conciencia por parte de personas heterosexuales aliadas de que sus identidades suelen ser cooptadas por la heteronormatividad y utilizadas en contra de sus amistades queer. Para evitar esto, algunas personas, tanto en la academia como en el activismo, han adoptado el término cisgénero como una manera de romper con esa situación. Según Schilt y Westbrook (2009):

Cis es el prefijo en latín que significa "en el mismo lado". Complementa a trans, el prefijo "a través de". "Cisgénero" sustituye los términos "no-transgénero" o "varón biológico" / "mujer biológica" para referirse a las personas cuyo sexo biológico, su cuerpo y su identidad personal coinciden. (pp. 119-120, énfasis en el original)

Este término busca eliminar la supremacía heterosexual en las definiciones de las personas, con el fin de adjudicar a cada ser humano, incluidas/os las y los heterosexuales, una característica particular. Si bien pone en evidencia las diferencias, no las juzga como "negativas" del modo en que lo hace el hecho que 
solo las personas "diferentes" deban ser identificadas por sus características particulares. Lo opuesto a cisgénero es transgénero.

\section{Transgeneridad}

De acuerdo con la teoría queer, las categorías de las identidades de género son múltiples y en constante cambio, con el fin de reflejar las diversas realidades que los individuos y las comunidades enfrentan en nuestro mundo en constante evolución. En muchos casos, la realidad de las personas no solo implica las cuestiones de orientación sexual, sino también las relacionadas con sus cuerpos, junto con nociones de identidad. Aquellas personas que transitan desde un lado del espectro del género hacia el otro — de varón a mujer o de mujer a varónson llamadas transgénero. Por supuesto, las transiciones pueden tomar varios caminos y no necesariamente esto implica un nuevo binarismo. Se prefiere el término transgénero en lugar del término transexual, ampliamente extendido en América Latina junto con el término travesti. De este último proviene el término traba como peyorativo de las mujeres transgénero en Argentina. En la comprensión de ambos términos, sigo a Mollenkott (2001), quien define esta distinción de la siguiente manera:

En principio, el término transgénero refiere sólo a las personas que han cambiado su sexo, pero no sus genitales-por ejemplo, un varón que utiliza estrógeno, vive como una mujer, pero no tiene planes de someterse a una cirugía de resignación de sexo. (hoy en día, esa persona se llamaría transexual no-operativo). Sin embargo, poco a poco, el término se ha ampliado para incluir intersexuales, transexuales, travestis, transformistas [drag queens/drag kings], andróginos, y cualquier persona que se siente "diferente" a los supuestos de la sociedad sobre el género. (p. 40)

Por otro lado, Shapiro (1991) también contribuye a explicar la noción de transgeneridad, que se usa para la designación de:

[...] quienes sienten que su verdadero género está en desacuerdo con su sexo biológico, más concretamente, designa a aquellas personas que están tratando de "pasar" como miembros del sexo opuesto, y, más concretamente, para designar a aquellas personas que o bien han tenido o se encuentran bajo tratamiento con miras a cambiar su sexo anatómico. (p. 249)

Lamentablemente, educadores, madres/padres y ministras/os, solo por nombrar algunos pocos actores sociales, generalmente no se preparan adecuadamente para responder a preguntas relacionadas con la vivencia de ser transgénero. El teólogo y ministro unitario universalista Greve (2006) - actual comoderador de la Asociación Unitaria Universalista de Estados Unidos - reflexiona sobre su trayectoria de vida en relación con este tema. Su testimonio ilustra muy bien la experiencia de cómo algunas personas no pueden comprender estas situaciones. 
Sin embargo, Greve (2006) es capaz de reflexionar sobre este asunto mucho más allá y desafiar las expectativas adjudicadas a la dicotomía de género:

Por desgracia, la necesidad de la sociedad por definir, dicotomizar, y límitar el género se basa en el sacrificio de la experiencia de la vida real de personas como yo. En lugar de confiar en nosotros para que identifiquemos nuestro propio género, la sociedad trata de obligarnos a una de las siguientes dos opciones: varón o mujer. Para mí hacer eso significaría negar una gran parte de quien soy. Aún cuando las personas transgénero son lo suficientemente fuertes como para rechazar la dicotomía del género, todavía estamos obligados a adquirir un continuo que pone dos opciones en extremos opuestos para que nosotros "transitemos" entre ambos: Antes yo era varón, ahora me estoy convirtiendo en una mujer o tú me identificas como mujer, pero ahora estoy creando el varón que siempre he sabido que soy. ¿Es tan difícil imaginar que hay más de 2 puntos fijos en esa intersección? ¿Qué pasa si hay un camino medio diferente entre ambos sexos? ¿Y qué pasa si hay personas que se quedan eternamente donde comenzaron, y otras transitan entre 2, 3 o más caminos alternativos, y si algunos viven en el medio de ese cruce donde todo se junta, y otros ni siquiera viven en lugares cerca de alguna intersección? (pp. 1-2)

En esta cita, Greve (2006) toca uno de los problemas básicos que las personas transgénero tienen que enfrentar: la dicotomía varón/mujer como mecanismo de segregación. En este sentido, la llamada transición que las personas transgénero enfrentan mientras buscan modificar su cuerpo se lee desde el modelo heteronormativo como una forma de normalizar el cuerpo anómalo de las personas transgénero. En otras palabras, un cuerpo equivocado se asimila como un cuerpo femenino o masculino correcto/normal. Aquí vemos un hecho más que notorio de parte del fascismo corporal (Córdova Quero, 2008) dentro del sistema heteropatriarcal, un hecho que ha sido incorporado en todas las áreas de la vida cotidiana y de las instituciones sociales.

Cabral (2005, citado por Zhang, 2005), uno de los activistas y académicos más importantes de la comunidad transgénero e intersexual en Argentina, ha analizado esta situación. En su discurso pronunciado en la reunión del 2005, de la Comisión de las Naciones Unidas para los Derechos Humanos, afirma lo siguiente:

En Argentina, por ejemplo, uno no puede ser declarado varón transexual a menos que uno se sienta atraído por las mujeres, es decir, todos los hombres trans deben ser primero lesbianas (basados en su sexo biológico) y heterosexuales (basados en su género elegido). Sin embargo, no todas las personas trans se identifican de tal manera. (Cabral, 2005, citado por Zhang, 2005)

En otras palabras, el proceso de normalización de los moldes del sistema heteropatriarcal y las normas de la performatividad de los cuerpos transexuales, la 
mayoría de las veces, los obliga a vivir una vida que no los representa. En esta misma línea, Berkins (2004), quien fuera una de las más importantes activistas de la comunidad transgénero e intersex en Argentina, declaraba lo siguiente:

Cuando renunciamos a ser el típico varón del sistema, el macho fuerte, dominador, entonces inmediatamente nos instalan en lo femenino. Pero no se trata de ser cualquier femenino; a lo dulce y divina se nos pide que le agreguemos una imagen y un cuerpo que sean lo más evidentes y exuberantes posible. Hay una exigencia que pasa por una puesta en escena remarcada, reforzada; cuanto más grande tengamos las tetas, la boca o la cola, mejor, puesto que esa femineidad, en nosotras, tiene que quedar completamente evidente. (p. 22)

Presionadas/os por la performatividad obligatoria, muchas personas transgénero hacen enormes esfuerzos para no mostrar su diferencia. El término utilizado para describir la experiencia de las personas transgénero es passing (pasar por) (Romero-Bachiller, 2003). Soy consciente de que este tema es muy problemático. Baso mi declaración en el trabajo de Shapiro (1991) quien, hablando de las personas transgénero, afirma lo siguiente:

Los esfuerzos de las personas transgénero para alcanzar su estado normal de género los involucra en lo que se conoce generalmente como "passing." Dado que el término "passing" tiene la connotación de ser aceptado por algo que no es, es importante tener en cuenta las complejidades que surgen cuando este término se aplica a lo que las personas transgénero están haciendo. En primer lugar, las personas transgénero frecuentemente creen que es cuando ellos están tratando de ejerciendo el papel de su sexo anatómico, a diferencia de su género subjetivamente experimentado, que están tratando de hacerse pasar por algo que no son. La forma en que con frecuencia ponen esto quiere decir que se sienten que están "disfrazados" como un varón o una mujer. Al mismo tiempo, las personas transgénero deben trabajar duro para pasar en su nueva condición de género, sin embargo, más auténtica que lo que ellos mismos creen que es. (p. 256)

En la mayoría de los casos, el proceso de normalización es una experiencia dolorosa que implica la erosión de la diferencia. Nuestras sociedades no están preparadas para hacer frente a las experiencias que no se ajustan a las categorías binarias. Las múltiples experiencias de las personas transgénero son muchas veces son esencialmente invisibilizadas. En mayo del 2013, el Congreso argentino aprobó la Ley 26.743 - también conocida como "Ley de Identidad de Género" - , con el fin de hacer justicia a las vidas de las personas transgénero. Esta ley básicamente da el derecho de las personas a obtener partidas de nacimiento y documentos de identidad con su género elegido sin necesidad de una orden judicial o una cirugía de reasignación de sexo. Esto refuerza situaciones que ya se venían dando en el país, como el caso del Consejo Superior de la Universidad Nacional de Córdoba, una de las universidades más antiguas de América Latina 
—fundada en 1613 - , que aprobó el 18 de octubre de 2011 una modificación de su funcionamiento, con el fin de adaptarse a la exigencia de documentos de clase y grado que concordaran con el género elegido por las personas transgénero. Lo que sucedió en Argentina fue motivado por la necesidad de respetar el género escogido de las personas transgénero y su derecho a ser respetadas/ os de acuerdo con él. Sin embargo, es un caso excepcional, porque las personas transgénero continúan enfrentando situaciones difíciles y hasta peligrosas en muchos lugares del planeta.

\section{Intersexualidad}

El término intersexual se utiliza para sustituir el término hermafrodita, que hoy en día se considera ambiguo, antiguo y peyorativo. Se refiere a las personas cuyo cuerpo biológico no solo es contrario a su identidad de género elegida, sino también en estricto sentido biológico, no se ajusta a las categorías "femenino/ masculino". Mollenkot (2001) contribuye a describir las principales categorías de intersexualidad como: a) Síndrome de Insensibilidad a los Andrógenos (sIA), también llamado Feminización Testicular; b) Síndrome de Insensibilidad Parcial a los Andrógenos; c) Virilización Progestina Inducida; d) Hiperplasia Suprarrenal Congénita, y e) Síndrome de Klinefelter (sk). Tradicionalmente, las personas intersexuales han sido forzadas a "cirugías de reasignación de sexo".

En los últimos años, muchas personas se han negado a someterse a estas cirugías afirmando que es una mutilación de sus cuerpos con el fin de ser aceptadas/ os dentro de la conformidad heteronormativa, es decir, una consecuencia del fascismo corporal:

Entiendo el fascismo corporal como la vigilancia, el control y la sanción de los cuerpos que adhieren a las construcciones hegemónicas de los cuerpos en la sociedad. Esta construcción es idealizada a fin de regir otro/s cuerpo/s que se consideran como "desviados" debido a su inadecuación a las construcciones hegemónicas del cuerpo. Esto está relacionado con cuestiones de discriminación y castigo hacia quienes resisten la (hetero)normalización corporal (Córdova Quero, 2008, pp. 83-84, énfasis en el original)

Los cuerpos cambian, son fluidos y son modificados por diferentes razones; y esas razones no se definen de maneras tajantes tanto en la esfera personal como social. Los cuerpos son, la mayor parte del tiempo, un intrincado balance entre ambas esferas, en diferentes grados o categorías. No podemos separar la identidad desplegada/elegida de su conexión con los cuerpos. En otras palabras, nuestros cuerpos son necesarios para nuestra forma de visualizarnos y mostrarnos a nosotras/os mismas/os en el mundo. Sin embargo, estos cuerpos no están obligados a performatividades fijas o estáticas. Más bien, varían de 
acuerdo con múltiples experiencias que también modificarán la forma en que las identidades se construyen.

Las personas intersexuales deben hacer frente a estos problemas a diario, y a veces sus vidas se tornan precarias debido a que el descubrimiento de su "insuficiencia" anatómica podría destruir sus vidas. La película XXY (2007), de la directora argentina Lucía Puenzo, describe gráficamente esta situación. En la historia, Alex, una adolescente intersexual de 15 años, con genitales masculinos y femeninos, se ve obligada a mudarse con sus padres desde Argentina a Uruguay. Aunque su madre busca que le realicen una cirugía de reasignación de género, Alex siente que ella no la desea. Alex conoce a Álvaro, el hijo de unos amigos de sus padres. Alex y Álvaro comienzan una relación en donde la condición intersexual de Alex no disminuye su amor. Sin embargo, tres chicos del pueblo acorralan a Alex y le sacan los pantalones en la playa y otra vez su "secreto" se da a conocer. Esto implica que tanto su vida como la de sus padres y su novio son perjudicadas por el estigma social y la discriminación asociados al tratar con alguien que es "diferente". La película captura el dilema cotidiano de las personas intersexuales y la de sus seres queridos en la vida real. Muestra de manera dolorosa las situaciones de discriminación que aún persisten.

Desde hace unos años, el 26 de octubre ha sido reconocido como el "Día Internacional de Concientización sobre la Intersexualidad".

\section{Conclusión}

Explorar los términos y la teoría queer como han sido expuestos en el presente escrito implica reconocer las complejas y diversas maneras de entender la conjunción entre sexualidad, identidad, género y teoría. Esos elementos no tienen valoración positiva ni negativa. Son parte de lo que la biodiversidad en el planeta ha dotado a todos los seres vivos. Debido a esto, la sexualidad y el género forman parte constitutiva de los seres humanos. Lo que ha sucedido es que las percepciones de determinadas personas o grupos comenzaron a catalogar la sexualidad o el género de acuerdo con otros intereses distintos. El problema nunca ha sido la sexualidad o el género en sí mismos, sino quiénes están habilitadas/os o no para ejercer determinado tipo de sexualidad o identidad de género. Lo que está detrás de las prohibiciones e invisibilizaciones de la historia de la diversidad sexo-genérica a lo largo del desarrollo de la humanidad son las concepciones e intereses particulares que sustentan esa invisibilización y la necesidad de control que conllevan tanto la sexualidad como el género en el contexto de la hegemonía del heteropatriarcado. Para desmantelar esa hegemonía necesitamos entonces abrir el abanico de posibilidades de los términos cooptados para ejercer el control heteropatriarcal. Este artículo es un aporte en la dirección de esa deconstrucción. 


\section{Referencias}

American Psychiatric Association (APA). (2014). Manual Diagnóstico y Estadístico de los Trastornos Mentales (DSM-5). Ciudad Autónoma de Buenos Aires: Editorial Panamericana.

Berkins, L. (2004). Eternamente atrapadas por el sexo. En J. Fernández, M. D ‘Uva \& P. Viturro (Eds.), Cuerpos ineludibles (pp. 19-24). Ciudad Autónoma de Buenos Aires: Ají de Pollo.

Brown, P. (1993). El cuerpo y la sociedad: los hombres, las mujeres y la renuncia sexual en el cristianismo primitivo. Barcelona: Muchnik.

Butler, J. (2002a). Cuerpos que importan: Sobre los límites materiales y discursivos del "sexo". Ciudad Autónoma de Buenos Aires: Paidós.

Butler, J. (2002b). Is Kinship Always Already Heterosexual. Differences: A Journal of Feminist Cultural Studies, 13(1), 14-44.

Butler, J. (2007). El género en disputa: el feminismo y la subversión de la identidad. Buenos Aires: Paidós.

Córdova Quero, H. (2008). This Body Trans/Forming Me: Indecencies in Transgender/Intersex Bodies, Body Fascism and the Doctrine of the Incarnation. En M. Althaus-Reid \& L. Isherwood (Eds.), Controversies in Body Theology (pp. 80-128). Londres: sсм Press.

Córdova Quero, H. (2010). Risky Affairs: Marcella Althaus-Reid Indecently Queering Juan Luis Segundo's Hermeneutic Circle Propositions. En L. Isherwood \& M. D. Jordan (Eds.), Dancing Theology in Fetish Boots: Essays in Honour of Marcella Althaus-Reid (pp. 207-218). Londres: scm Press.

Córdova Quero, H. (2011). Sexualizando la Trinidad: Aportes desde una teología de la liberación queer a la compresión del misterio divino. Cuadernos de Teología, 30, 53-70.

Córdova Quero, H. (2013). Teo-queer-nautas: Teologías queer explorando nuevos horizontes en el siglo xxi. En S. R. Lima da Silva, G. Boehler \& L. Bedurke (Eds.), Teorías queer y teologías: Estar... en otro lugar (pp. 97-135). San José, Costa Rica: Departamento Ecuménico.

Córdova Quero, H. (2016). Embodied (Dis)Placements: The Intersections of Gender, Sexuality, and Religion in Migration Studies. En E. FiddianQasmiyeh, J. B. Saunders \& S. Snyder (Eds.), Shifts in the Sacred: Crossing Borders in Religion and Global Migrations (Religion and Global Migrations series \#1) (pp. 151-171). Nueva York: Palgrave MacMillan. 
Davis, L. J. (1997). Constructing Normalcy: The Bell Curve, the Nobel, and the Invention of the Disabled Body in the Nineteenth Century. En L. J. Davis (Ed.), The Disability Studies Reader (pp. 3-16). Nueva York: Routledge.

Delany, S. R. (1999). Shorter Views: Queer Thoughts \& the Politics of the Paraliterary. Hanover: Wesleyan University.

De Lauretis, T. (1991). Queer Theory: Lesbian and Gay Sexualities. Differences: A Journal of Feminist Cultural Studies, 3(2), iii-xviii.

Drescher, J. (2015). Out of Dsm: Depathologizing Homosexuality. Behavioral Sciences, 5(4), 565-575. Dor: https://dx.doi.org/10.3390\%2Fbs5040565

Elliott, A. (2003). Concepts of the Self, Serie "Key Concepts". Cambridge: Polity Press.

Foucault, M. (1998). Historia de la sexualidad, Volumen 1: La voluntad de saber. Ciudad de México: Siglo XXI.

Foucault, M. (2002). Vigilar y castigar: el nacimiento de la prisión. Buenos Aires: Siglo XXI.

Franke-Watson, M. B. (1999). Sexual Division of Labor. En H. Tierney (Ed.), Women's Studies Encyclopedia, Volumen 3 (pp. 1299-1300). Westport: Greenwood Press.

Goffman, E. (1986). Stigma: Notes on the Management of Spoiled Identity. Nueva York: Simon \& Schuster.

Greve, B. (2006). Courage from Necessity. Sermón predicado en la Iglesia de la Comunidad de Todas las Personas, Iglesia Unitaria Universalista, San Francisco, California, el 3 de junio.

Haldeman, D. C. (1994). The Practice and Ethics of Sexual Orientation Conversion Therapy. Journal of Consulting and Clinical Psychology, 62(2), 221-227. Dor: https://doi.org/10.1037//0022-006x.62.2.221

Halperin, D. M. (2003). The Normalization of Queer Theory. Journal of Homosexuality 45(2-4), 339-343. Dor: https://doi.org/10.1300/J082v45n02_17

Indra, D. (2004). Not a 'Room of One's Own': Engendering Forced Migration Knowledge and Practice. En D. Indra (Ed.), Engendering Forced Migration: Theory and Practice (pp. 1-22). Nueva York: Berghahn Books.

Irigaray, L. (1985). This Sex Which is not One. Nueva York: Cornell University.

Jasose, A. (1996). Queer Theory: An Introduction. Nueva York: New York University Press. 
Jung, P. B., \& Smith, R. F. (1993). Heterosexism: An Ethical Challenge. Nueva York, NY: State University of New York.

Kemp, J. (2009). Queer Past, Queer Present, Queer Future. Graduate Journal of Social Science, 6(Número especial 1), 3-23.

Marinucci, M. (2010). Feminism is Queer: The Intimate Connection Between Queer and Feminist Theory. Londres: Zed Books.

Mollenkott, V. R. (2001). Omnigender: A Trans-Religious Approach. Cleveland: The Pilgrim Press.

Nussbaum, M. C. (1997). Constructing love, desire, and care. En D. M. Estlund \& M. C. Nussbaum (Eds.), Sex, Preference, and Family: Essays on Law and Nature (pp. 17-43). Oxford: Oxford University.

Puenzo, L. (dir.) (2007). XXY, 86 minutos, Film Movement, DvD.

Ricouer, P. (1994). Fe y filosofía: Problemas del lenguaje religioso. Ciudad Autónoma de Buenos Aires: Prometeo.

Romero-Bachiller, C. (2003). De diferencias, jerarquizaciones excluyentes, y materialidades de lo cultural. Una aproximación a la precariedad desde el feminismo y la teoría queer. Cuadernos de Relaciones Laborales, 21(1), 33-60.

Schidlo, A., \& Schroeder, M. (2002). Changing Sexual Orientation: A Consumer's Report. Professional Psychology: Research and Practice, 33(3), 249-259. DoI: https://doi.org/10.1037/0735-7028.33.3.249

Schilt, K., \& Westbrook, L. (2009). Doing Gender, Doing Heteronormativity: 'Gender Normals,' Transgender People, and the Social Maintenance of Heterosexuality. Gender \& Society, 23(4), 440-464. Dor: https://doi. org/10.1177/0891243209340034

Sedwick, E. K. (1998). Epistemology of the Closet. Berkeley: University of California.

Shapiro, J. (1991). Transsexualism: Reflections on the Persistence of Gender and the Mutability of Sex. En J. Epstein \& K. Straub (Eds.), Body Guards: The Cultural Politics of Gender Ambiguity (pp. 248-279). Londres: Routledge.

Sterba, J. P. (1997). Racism and Sexism: The Common Ground. En N. Zack (Ed.), Race/Sex: Their Sameness, Difference, and Interplay (pp. 61-71). Nueva York: Routledge.

Sudha, D. K. (2000). Gender Roles. Nueva Delhi: ApH Publishing. 
Ticktin, M. (2008). Sexual Violence as the Language of Border Control: Where French Feminist and Anti-immigrant Rhetoric Meet. Signs: Journal of Women in Culture and Society, 33(4), 863-889. Dor: https://doi.org/10.1086/528851

Yamanaka, K. (2003). Feminized Migration, Community Activism and Grassroots Transnationalisation in Japan. Asian and Pacific Migration Journal, 12(1-2), 155-188. Dor: https://doi.org/10.1177/011719680301200107

Zhang, S. (2005). Gender Rights are Human Rights. Report on the Discussion Panel on Trangender/ Intersex Issues at the 65th Session of the Commission on Human Rights of the United Nations, April 13. 